OPEN ACCESS

Edited by:

Takatsugu Ishimoto,

Kumamoto University, Japan

Reviewed by:

Kazumichi Kawakubo,

Hokkaido University Hospital, Japan

Tatsunori Miyata,

Cleveland Clinic, United States

*Correspondence:

Kiyoshi Hasegawa

kihase-tky@umin.ac.jp

Yasuteru Urano

uranokun@m.u-tokyo.ac.jp

Specialty section:

This article was submitted to

Gastrointestinal Cancers,

a section of the journal

Frontiers in Oncology

Received: 25 May 2021 Accepted: 04 August 2021 Published: 19 August 2021

Citation:

Takahashi R, Ishizawa T, Sato $M$

Inagaki Y, Takanka M, Kuriki Y,

Kamiya M, Ushiku T, Urano $Y$ and Hasegawa K (2021) Fluorescence

Imaging Using Enzyme-Activatable

Probes for Real-Time Identification of

Pancreatic Cancer.

Front. Oncol. 11:714527.

doi: 10.3389/fonc.2021.714527

\section{Fluorescence Imaging Using Enzyme-Activatable Probes for Real-Time Identification of Pancreatic Cancer}

\author{
Ryugen Takahashi ${ }^{1}$, Takeaki Ishizawa ${ }^{1}$, Masumitsu Sato ${ }^{1}$, Yoshinori Inagaki ${ }^{1}$, \\ Mariko Takanka ${ }^{2}$, Yugo Kuriki ${ }^{3}$, Mako Kamiya ${ }^{4}$, Tetsuo Ushiku ${ }^{2}$, Yasuteru Urano ${ }^{3,4 *}$ \\ and Kiyoshi Hasegawa ${ }^{1 *}$

\footnotetext{
1 Hepato-Biliary-Pancreatic Surgery Division, Department of Surgery, Graduate School of Medicine, University of Tokyo, Tokyo, Japan, 2 Department of Pathology, Graduate School of Medicine, The University of Tokyo, Tokyo, Japan,

${ }^{3}$ Laboratory of Chemistry and Biology, Graduate School of Pharmaceutical Sciences, The University of Tokyo, of Tokyo, Tokyo, Japan
} \\ Tokyo, Japan, ${ }^{4}$ Laboratory of Chemical Biology and Molecular Imaging, Graduate School of Medicine, The University
}

Introduction: Radical resection is the only curative treatment for pancreatic cancer, which is a life-threatening disease. However, it is often not easy to accurately identify the extent of the tumor before and during surgery. Here we describe the development of a novel method to detect pancreatic tumors using a tumor-specific enzyme-activatable fluorescence probe.

Methods: Tumor and non-tumor lysate or small specimen collected from the resected specimen were selected to serve as the most appropriate fluorescence probe to distinguish cancer tissues from noncancerous tissues. The selected probe was sprayed onto the cut surface of the resected specimen of cancer tissue to acquire a fluorescence image. Next, we evaluated the ability of the probe to detect the tumor and calculated the tumor-to-background ratio (TBR) by comparing the fluorescence image with the pathological extent of the tumor. Finally, we searched for a tumor-specific enzyme that optimally activates the selected probe.

Results: Using a library comprising 309 unique fluorescence probes, we selected GPHMRG as the most appropriate activatable fluorescence probe. We obtained eight fluorescence images of resected specimens, among which four approximated the pathological findings of the tumor, which achieved the highest TBR. Finally, dipeptidylpeptidase IV (DPP-IV) or a DPP-IV-like enzyme was identified as the target enzyme.

Conclusion: This novel method may enable rapid and real-time visualization of pancreatic cancer through the enzymatic activities of cancer tissues.

Keywords: pancreatic cancer, pancreatectomy, fluorescence imaging, activatable probe, intraoperative diagnosis, dipeptidyl peptidase-IV (DPP-IV) 


\section{INTRODUCTION}

Pancreatic cancer is a major life-threatening disease (1-4). Despite recent advances in chemotherapy and radiotherapy, complete resection remains the only curative treatment (5-7). However, it is often difficult to accurately identify the boundaries of cancer tissues during surgery, which may lead to incomplete removal of cancer tissues and unfavorable postoperative survival $(6,7)$. For patients administered preoperative chemo(radio) therapy $(8,9)$, it is particularly difficult to identify viable cancer tissues, even in pathological examinations of resected specimens (10).

In 2011, Urano et al. reported a novel fluorescence imaging technique using an "activatable" probe, which is initially nonfluorescent but emits fluorescence immediately after its hydrolysis by $\gamma$-glutamyltranspeptidase overexpressed specifically in various cancer cells (11). Subsequently, more than 400 activatable fluorescence probes comprising amino acid or sugar residues that serve as a reactive moiety and target the aminopeptidase or glucosidase have been developed. Furthermore, fluorescently activatable scaffolds such as hydroxymethyl rhodamine-green (HMRG) or hydroxymethyl rhodol with trifluoroethyl group (HMRef) were developed (12), enabling visualization of breast cancer (12-14), esophageal cancer $(15,16)$, liver cancer (17), lung cancer (18), head and neck cancer $(19,20)$, colorectal cancer (21), thyroid cancer (22), and glioblastoma (23). Regarding real-time imaging of pancreatic cancer, other approaches using activatable probes can be indicated (24-26), albeit applications of these techniques to fresh human samples have not yet been reported. Here we searched for activatable fluorescence probes for real-time identification of viable pancreatic cancer tissues in resected specimens.

\section{MATERIAL AND METHODS}

The Institutional Review Board of the University of Tokyo Hospital approved this study [IRB No. 2957- (11)].

\section{Sample Collection}

Fresh tissue samples were collected from resected specimens of patients who underwent radical pancreatectomy for pancreatic adenocarcinoma from April 2017 to December 2020. Written informed consent was obtained from all patients. For the primary and secondary probe selection, $3-5 \mathrm{~mm}$-in-size tissue fragments were obtained from obvious cancerous regions and non-cancerous pancreatic parenchyma with confirmation by the pathologist (M.T.), just after the removal of pancreatic specimens.

\section{Primary Probe Selection}

In this study, totally 309 dipeptides-HMRG fluorescence probes were used from our probe library. The concept and synthetic methods of these probes have been described elsewhere (12). Briefly, these probes were synthesized by placing an amino-acid residue selected from 21 amino-acids at P1 and P2 position of $\mathrm{Xaa}(\mathrm{P} 2)-\mathrm{Yaa}(\mathrm{P} 1)-H M R G$ (Supplementary Table 1). Among the chemically stable compounds, candidate fluorescence probes were first selected using lysates prepared from cancer and noncancerous tissue samples $(12,15,23)$. Briefly, the tissue cut by scissors were homogenized with $1.0 \mathrm{~mL}$ of T-per tissue protein in a Lysing Matrix D. After the centrifuge $(1,000$ rpm $\times 5 \mathrm{~min}$ at $4^{\circ} \mathrm{C}$ ), the supernatant was collected as the lysate. Then, $5 \mu \mathrm{L}$ of the lysate $(0.20 \mathrm{mg} / \mathrm{mL}$ protein $)$ was added to the wells of a black 384-well plate, each containing $15 \mu \mathrm{L}$ of each candidate probe from a library of dipeptide-HMRG compounds (23). The final concentrations of a candidate probe and lysate protein were $1.0 \mu \mathrm{M}$ and $0.050 \mathrm{mg} / \mathrm{dL}$, respectively. The fluorescence intensity (FI) of each sample was measured using an Envision Multilabel Plate Reader (PerkinElmer, Massachusetts, USA) 0-60 min after the addition of lysates at $37^{\circ} \mathrm{C}$. The excitation and emission wavelengths were $485 \mathrm{~nm}$ and $535 \mathrm{~nm}$, respectively. The increase of FI was calculated as follows:

$$
(\text { FI increase })=(\text { FI at } 60 \mathrm{~min})-(\text { FI at } 0 \mathrm{~min})
$$

Then, we calculated the difference and the ratio of FI increase between cancer and noncancer lysates, and the probes of which difference or ratio represented the $\geq 90$ th percentile of all probes was subjected to subsequent evaluations.

\section{Secondary Probe Selection}

Candidate fluorescence probes were sprayed directly onto a few millimeters of cancer tissues and noncancerous tissues placed in an eight-well plate. The cancerous and noncancerous tissues were collected as the same way as the primary probe selection, and divided into smaller specimen by scissors respectively. When the size of original tissue samples was insufficient for creating 6 fragments, fluorescence imaging was performed prioritizing candidate probes with better outcomes in the primary screening. The concentration and volume of each fluorescence probe was $50 \mu \mathrm{M}$ and $200 \mu \mathrm{L}$, respectively. Images of fluorescence were obtained using the Maestro in Vivo Imaging System (PerkinElmer, Massachusetts, USA), with the blue filter settings (excitation and emission wavelengths of 435-480 nm and $490 \mathrm{~nm}$ long pass), respectively, acquired 0 (before), 1, 3, 5, $10,15,20,25$, and $30 \mathrm{~min}$ after the administration of fluorescence probes. FI was calculated by subtracting the average in the region of interest (ROI) at $1 \mathrm{~min}$ from that at $30 \mathrm{~min}$, according to the fluorescence images extracted at $540 \mathrm{~nm}$. Finally, candidate fluorescence probes were refined according to the difference in contrast of FI between cancer tissues and noncancerous tissues.

\section{Macroscopic Evaluation of Cancer Using Whole Surgical Specimens}

Immediately after pancreatic resection, the whole specimen was cut to include the maximum diameter of pancreatic cancer tissues. The selected florescence probe ( $4 \mathrm{~mL}, 50 \mu \mathrm{M}$ solution) was sprayed directly onto the cut surfaces, followed by fluorescence imaging using the Maestro in Vivo imaging System, as described above. The accuracy of fluorescence imaging to delineate pancreatic cancer was evaluated by a surgeon (R.T.) and a pathologist (M.T.) with reference to histopathological findings of the same planes. The tumor-to- 
background ratio (TBR) was calculated as the increase in the mean FI from $1 \mathrm{~min}$ to $30 \mathrm{~min}$ after administration of the probe to cancer tissues and noncancerous pancreatic tissues, as follows:

$$
T B R=\frac{\text { FI increase of the cancerous tissue }}{\text { FI increase of the non - cancerous tissue }}
$$

The data were obtained using the Maestro In Vivo Imaging System, described above, according to macro- and microscopic pathological findings of the cut surfaces and their corresponding fluorescence images.

\section{Exploration of Target Enzymes}

Through the probe screening process, dipeptidyl peptidases were suspected as target enzymes that may be overexpressed specifically in pancreatic cancer tissues. Thus, we first confirmed the ability of DPP-IV and related enzymes to activate candidate fluorescence probes by measuring changes of FI for 1,000 seconds after the addition of human recombinant DPP-IV (0.040 units; D4943, Sigma-Aldrich), DPP-VIII (1.0 $\mu \mathrm{g}$; ab162872, abcam), or DPP-IX (1.0 $\mu \mathrm{g}$; ab79621, abcam) to 3.0 $\mathrm{mL}$ of probe $(1.0 \mu \mathrm{M})$ using the F-7000 Hitachi Fluorescence Spectrophotometer (Hitachi, Tokyo, Japan). The excitation and emission wavelengths were $495 \mathrm{~nm}$ and $525 \mathrm{~nm}$, respectively. FI in the cancer tissues used in the secondary screening were also measured after administration of DPP-IV inhibitor (K579, CalbioChem) at a dose of $100 \mu \mathrm{M}$. Finally, the expression of DPP-IV on cut surfaces of whole surgical specimens was evaluated using immunohistochemical (IHC) analysis with an anti-DPP-IV mouse monoclonal antibody (TA500733; Origene Technologies Inc, Rockville, MD). Antigen retrieval was performed at $110^{\circ} \mathrm{C}$ for $15 \mathrm{~min}$. The anti-DPP-IV antibody was diluted 1:100, and the tissues were incubated overnight at $4^{\circ} \mathrm{C}$. The IHC results were evaluated by a pathologist (M.T.) uninformed about the outcomes of fluorescence imaging.

\section{RESULTS}

\section{Primary and Secondary Probe Selection}

Primary selection of fluorescence probes employed lysates prepared from five resected specimens, leading to the identification of candidate probes from 309 fluorescence probes (Supplementary Table 1). When the differences and ratios of FI increase between cancer and non-cancer lysates were calculated (Supplementary Table 2), 14 out of the 19 HMRG-based fluorescence probes with dipeptides with a prolyl residue at the $\mathrm{P} 1$ position (XaaP-HMRG) ranked in the upper 90 percentile (Figure 1A). Based not only on the FI differences/ratios but also probe stability and the absolute values of FI increase in non-cancer lysates, which could decrease cancer detectability on tissue samples, AcKP(Acetylated Lysine-Proline-), EP- (Glutamate-Proline-), GP(Glycine-Proline-), LP- (Leucine-Proline-), PP (ProlineProline), and YP-HMRG (Tyrosine-Proline-HMRG) were selected in this study (Figure 1B) for the second screening using fresh tissue fragments obtained from 11 patients with pancreatic adenocarcinoma. As a result, fluorescence imaging using GP-HMRG yielded the highest intensity differences in FI after 30 min between cancer and noncancerous tissues [median (range), 3.49 (1.03-8.11) a.u. vs 1.12 (0.42-2.09) a.u., $\mathrm{P}=0.002$; Wilcoxon's rank-sum test] (Figures 1C, D). GP-HMRG was therefore selected to evaluate fluorescence imaging to specifically detect cancer tissues in whole surgical specimens. Demographic background of the totally 16 patients who provided lysates or tissue samples were demonstrated in Supplementary Table 3.

\section{Fluorescence Imaging of Whole Surgical Specimens Using GP-HMRG}

Detection of cancer tissues using fluorescence imaging was evaluated by spraying GP-HMRG onto cut surfaces of whole surgical specimens immediately after resection of eight patients with pancreatic adenocarcinoma. Patients' demographic characteristics are summarized in Table 1. Neoadjuvant chemotherapy with gemcitabine and nab-paclitaxel was indicated to two patients who underwent surgery. Three patients were treated for diabetes mellitus but were not preoperatively administered DPP-IV inhibitors.

The median TBR of the fluorescence images following the administration of GP-HMRG was 1.96 (range, 1.13-3.44). In five patients with TBRs ranging from 1.93 to 3.44 , fluorescence signals in cancer tissues were nearly homogenous and grossly discriminable from the surrounding pancreatic tissues (Figure 2). In the remaining three patients, including two who underwent preoperative chemotherapy, cancer tissues emitted heterogenous fluorescence signals, making it difficult to discriminate them from noncancer tissues (Figure 3). Fluorescence imaging identified a significant signal increase (TBR, 2.04) in the connective tissues surrounding the splenic artery of one patient in the latter group, which pathological examination subsequently diagnosed as perineural and lymphatic infiltration by viable cancer cells, while the main tumor included fibrosis and mucinous changes with a few viable cancer cells (TBR, 1.13), likely caused by preoperative chemotherapy (Figure 4).

\section{Identification of Target Enzymes That Activate GP-HMRG}

The in vitro fluorescence spectrum of GP-HMRG after adding DPPs indicated that the probe was converted to highly fluorescent HMRG upon reaction with DPP-IV and DPP-IX (Figure 5). On the cancer tissue specimens used in the secondary screening (available in 4 cases), FI increase was markedly suppressed when GP-HMRG was administered with the inhibitor (Figure 6). In contrast, IHC analysis of the resected specimens of eight patients did not detect an unambiguous difference in the expression levels of DPP-IV between cancer and surrounding pancreatic tissues (Figures 2-4).

\section{DISCUSSION}

Here we screened GP-HMRG, among 309 candidates of activatable fluorescence probes, for its ability to specifically 


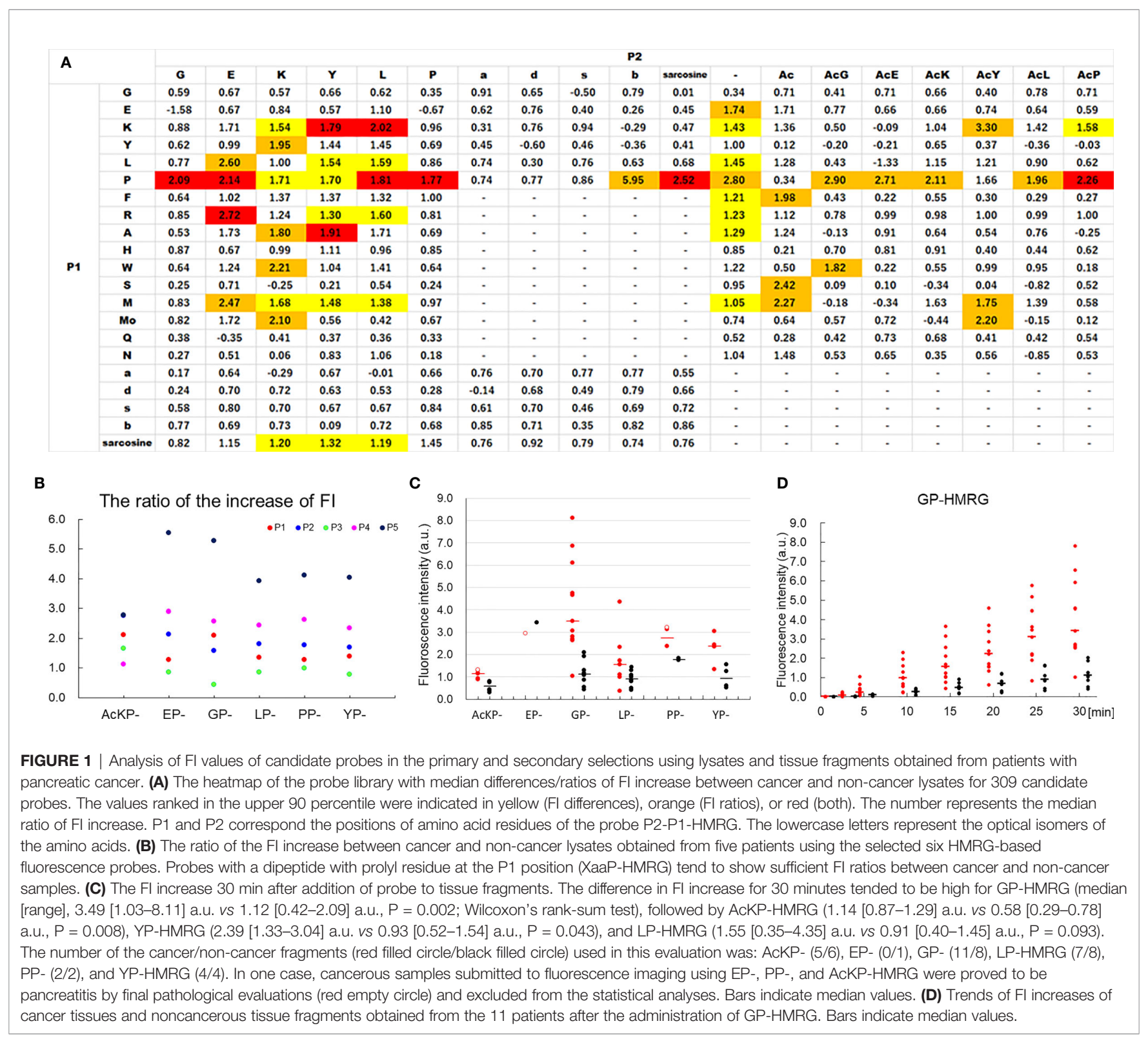

TABLE 1 | Patients' demographic characteristics and outcomes of fluorescence imaging using GP-HMRG.

\begin{tabular}{|c|c|c|c|c|c|c|c|c|}
\hline Patient & Age (y) & Sex & DM & NAC, (effect*) & $\begin{array}{l}\text { Preoperative } \\
\text { CA19-9 (IU/mL) }\end{array}$ & $\begin{array}{c}\text { Surgical } \\
\text { procedures }\end{array}$ & Histological type & TBR of $\mathrm{FI}$ \\
\hline 1 & 70 & $M$ & - & - & 68 & DP & Adenosquamous & 3.44 \\
\hline 4 & 74 & M & - & - & 39 & DP & tub2 > tub1 & 1.98 \\
\hline 5 & 73 & $\mathrm{~F}$ & + & - & 1 & DP & tub1 > tub2 & 1.93 \\
\hline 6 & 68 & $M$ & + & - & 393 & $\mathrm{PD}$ & tub2 > tub1 & 1.47 \\
\hline
\end{tabular}

$D M$, diabetic mellitus; NAC, neoadjuvant chemotherapy; PD, pancreaticoduodenectomy; DP, distal pancreatectomy; DP-CAR, distal pancreatectomy with celiac axis resection; TBR, tumor-to-background ratio; tub1/tub2, well/moderately differentiated tubular adenocarcinoma; por, poorly differentiated adenocarcinoma.

*Evans classification.

**With perineural and lymphatic infiltration of viable cancer cells to the splenic artery. 


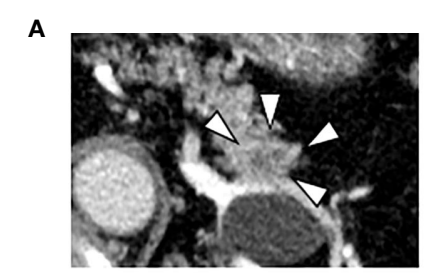

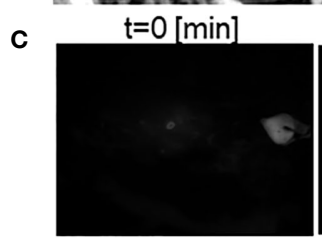

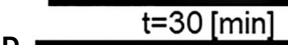

D

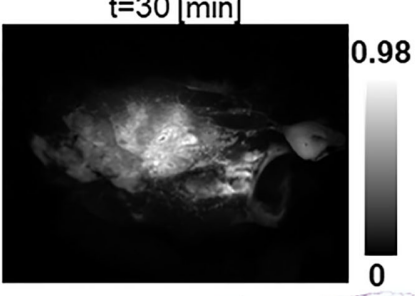

$\mathbf{F}$

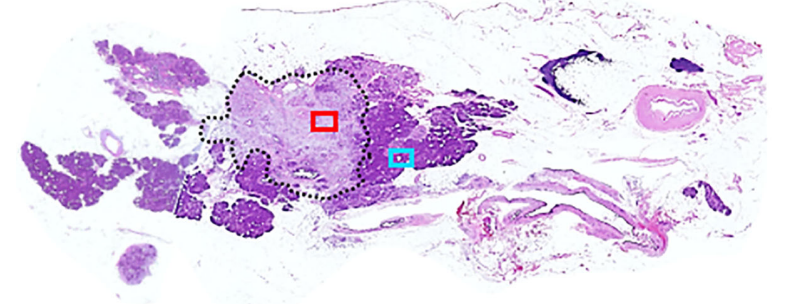

$\mathrm{t}=1$ [min]

Pseudo-real color
B
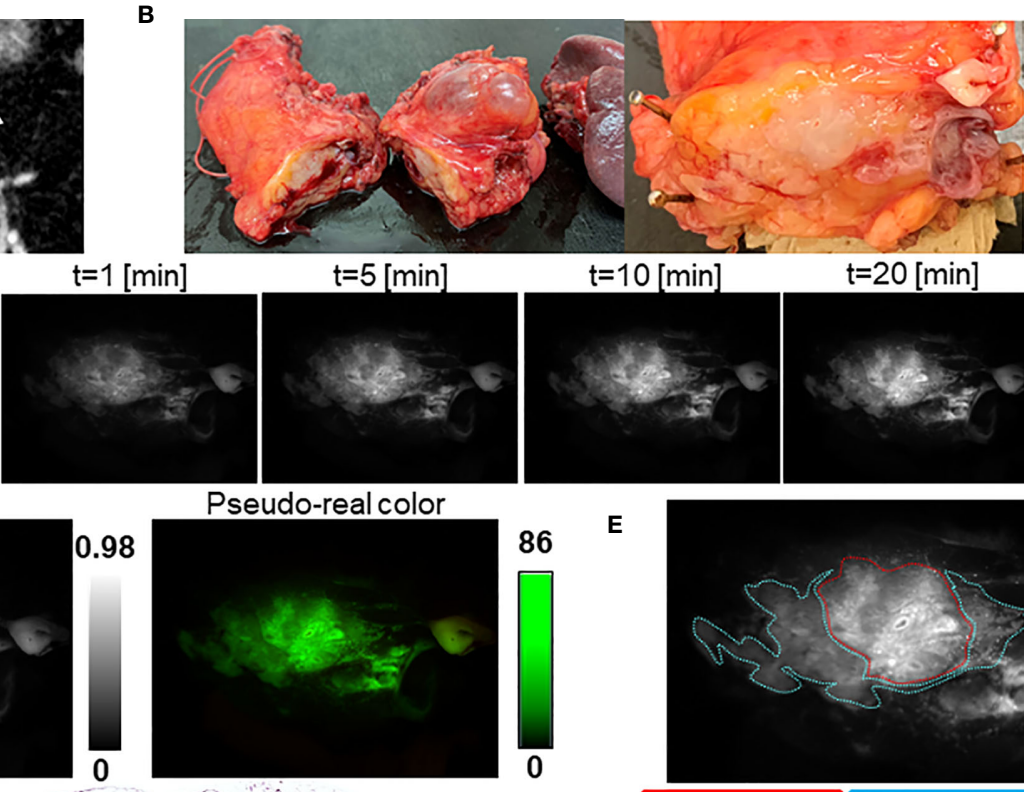

86

E

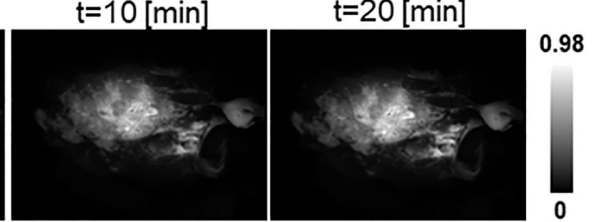

IHC

(DPP-IV)

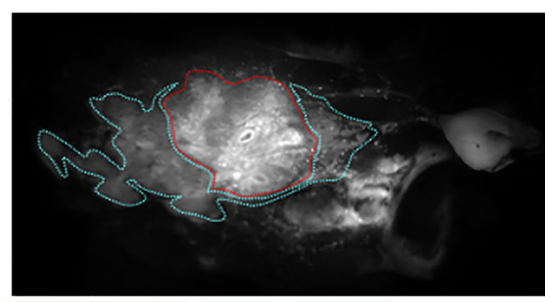

HE
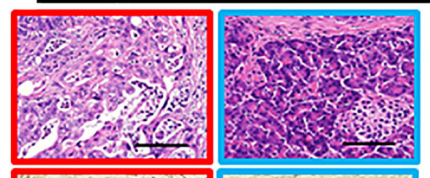

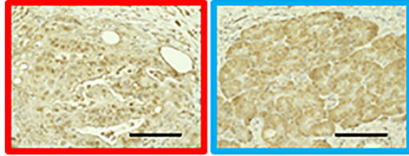

FIGURE 2 | Fluorescence imaging using GP-HMRG to analyze whole surgical specimens demonstrated homogenous increases in fluorescence signals emitted by pancreatic cancer tissues (Patient No. 2). (A) Preoperative contrast-enhanced computed tomography of pancreatic tail cancer (arrowheads). (B) Macroscopic image of the DP specimen after making cut surfaces of the tumor in the operation room (left). Right indicates magnified view of the cut surface including the tumor. (C) Increase of fluorescence signals after spraying GP-HMRG on the cut surface. (D) Fluorescence image (left) and its pseudo-real color image (right) of the cut surface 30 min after probe administration. (E) Relationships between fluorescence signals and distributions of cancer tissues (red dotted line) and surrounding pancreatic tissues (blue dotted line) according to histological findings. (F) Low-magnification histopathological image of hematoxylin-eosin (H\&E) staining corresponding to fluorescence images (left, dotted line indicates cancer boundaries). Magnified views of H\&E staining and IHC analysis of DPP-IV in cancer (red) and pancreatic (blue) tissues (right). Scale bar $=100 \mu \mathrm{m}$.

identify pancreatic cancer tissues. Fluorescence imaging using GP-HMRG sprayed onto cut surfaces of fresh resected specimens visualized cancer tissues as homogenous fluorescing regions with a high (>1.9) TBR in five of eight patients. Among the remaining three patients, fluorescence signals in cancer tissues were heterogenous and therefore insufficient for unambiguous discrimination from surrounding noncancerous tissues. However, in one patient who underwent preoperative chemotherapy, fluorescence imaging visualized grosslyunidentifiable cancer infiltrations around the splenic artery. These results suggest that fluorescence imaging using GPHMRG potentially visualizes the spread of pancreatic cancer cells in real time, which may be useful for intraoperative diagnosis of surgical margins as well as for preoperative endoscopic evaluations of intraductal lesions.

The major advantage of using activatable probes is their ability to rapidly identify in real-time cancer tissues according to enzymatic activities, which are specifically expressed by cancer cells. Moreover, in the present series, an increase in fluorescence signals emitted by cancer tissues was identified 1 min after the topical administration of GP-HMRG. The FI values of cancer tissues in the remaining three patients may be decreased because of fewer viable cancer cells with fibrosis and mucinous changes, likely caused by preoperative chemotherapy.

Recently developed fluorescence imaging techniques for intraoperative identification of pancreatic cancer employ 5aminolevulinic acid (27), indocyanine green (28), novel fluorophores targeting carbohydrate antigen 19-9 (CA19-9) (29), carcinoembryonic antigen (CEA) (30-35), epidermal growth factor receptor (EGFR) $(36,37)$, and insulin-like growth factor 1 receptor (IGF-1R) (38). However, these techniques, most of which involve systemic administration of "non-activatable" probes, usually require longer intervals for washout of fluorescence agents from background tissues, which may lead to lower TBRs compared with those of activatable probes topically administered during surgery. 

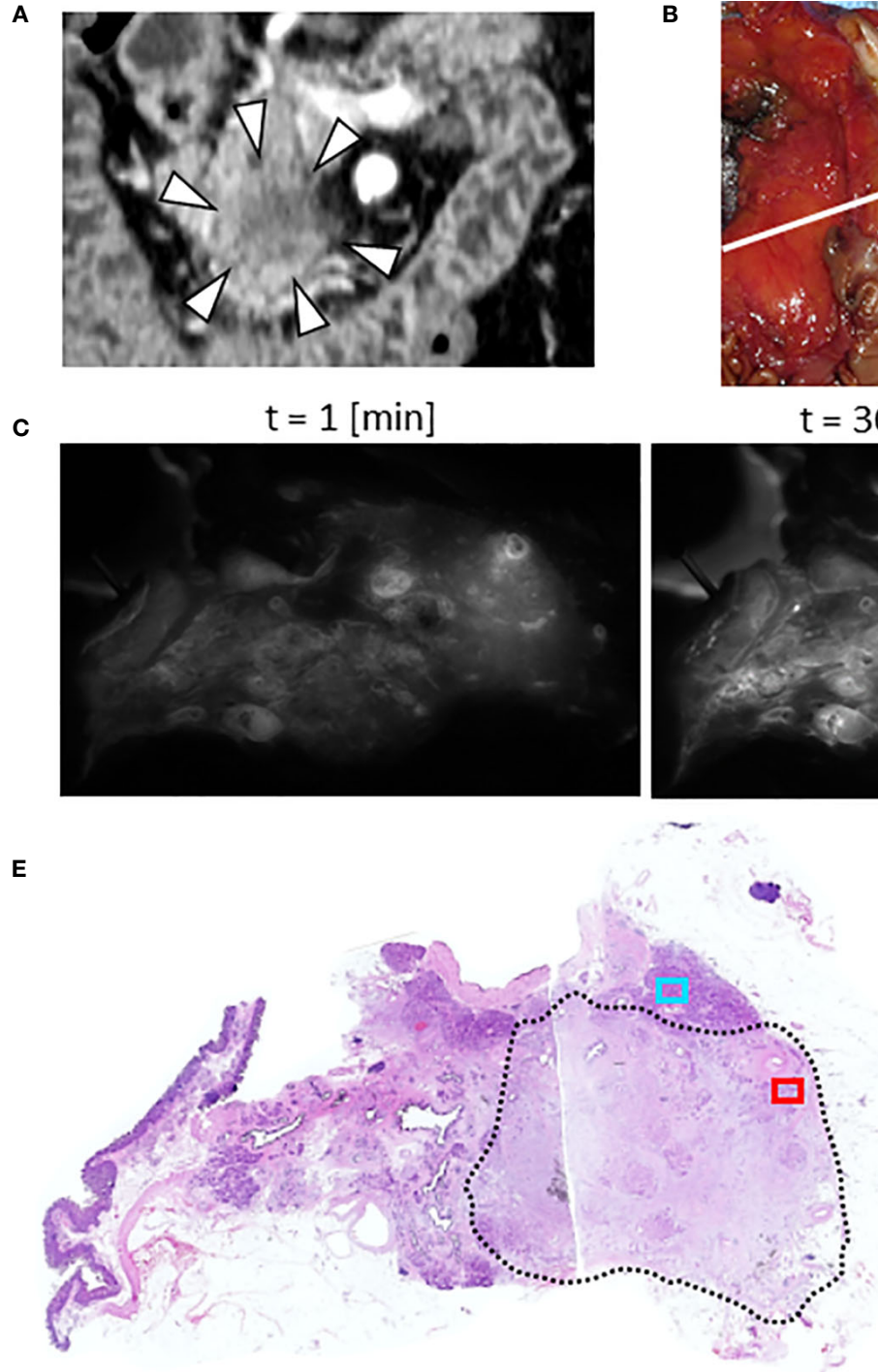

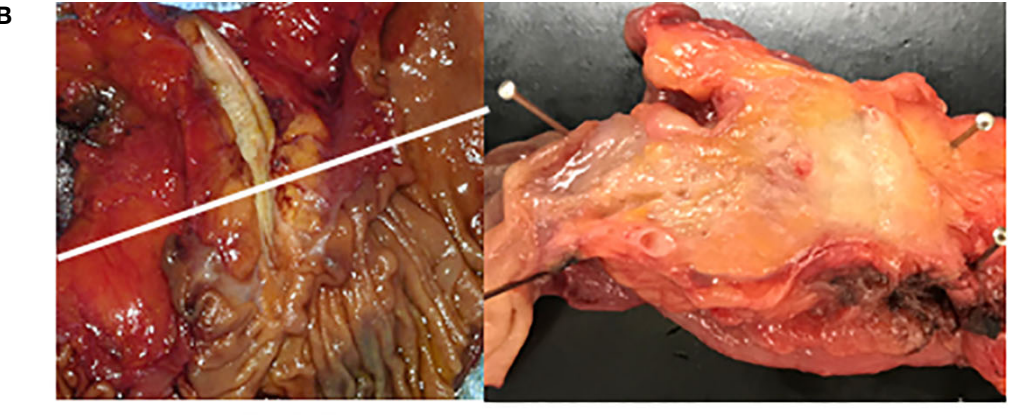

$\mathrm{t}=30[\mathrm{~min}]$

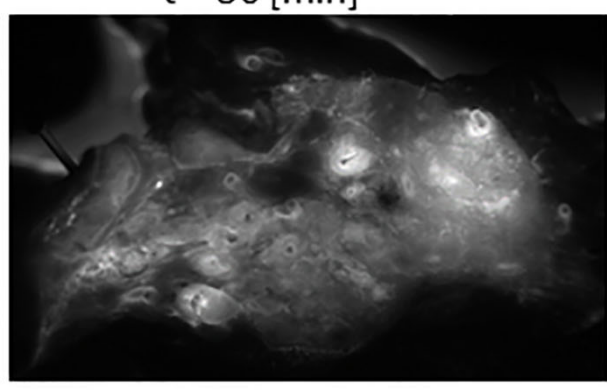

D

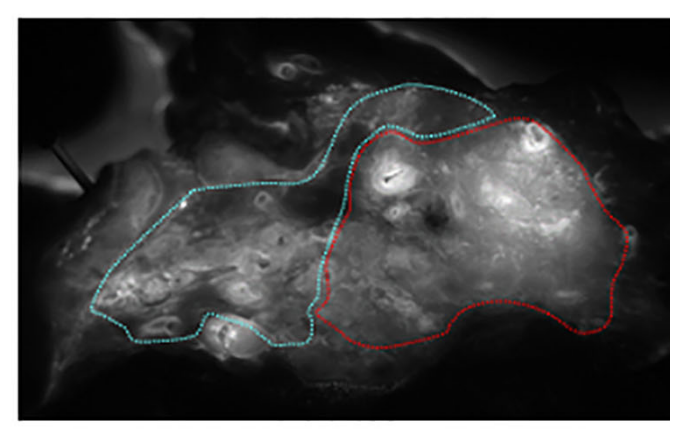

0.48

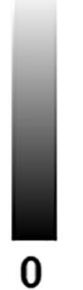

0

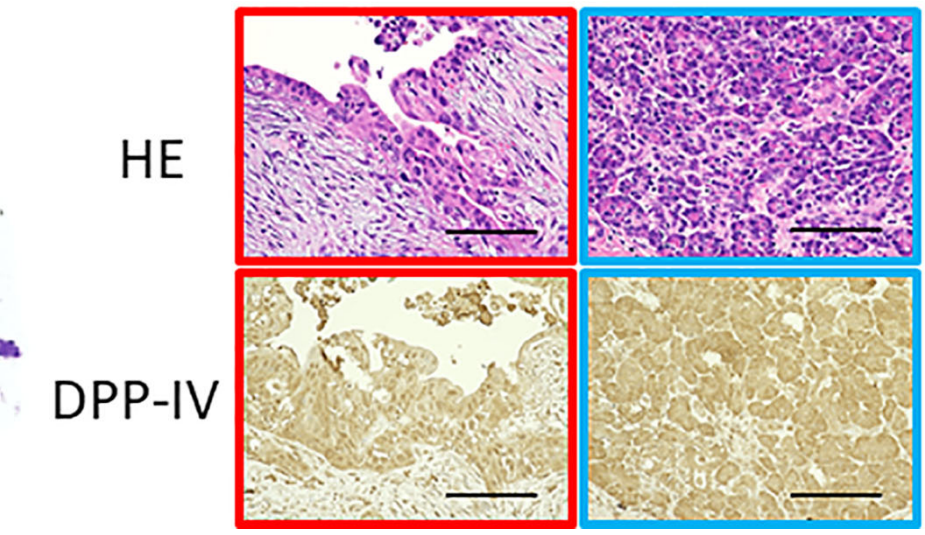

FIGURE 3 | Fluorescence imaging using GP-HMRG to probe whole surgical specimens demonstrates heterogenous increases in fluorescence signals emitted by pancreatic cancer tissues (Patient No. 6). (A) Preoperative contrast-enhanced computed tomography of pancreatic head cancer (arrowheads). (B) Macroscopic image of the PD specimen (left) and cut surface along the white line including the tumor (right). (C) Increase of fluorescence signals after spraying GP-HMRG on the cut surface. (D) Relationships between fluorescence signals and distributions of cancer tissues (red dotted line) and surrounding pancreatic tissues (blue dotted line) according to histological findings. (E) Low-magnification histopathological image of H\&E staining corresponding to fluorescence images (left, dotted line indicates cancer boundaries).

Magnified views of H\&E staining and IHC analysis of DPP-IV in cancer (red) and pancreatic (blue) tissues (right). Scale bar $=100 \mu \mathrm{m}$. 
A

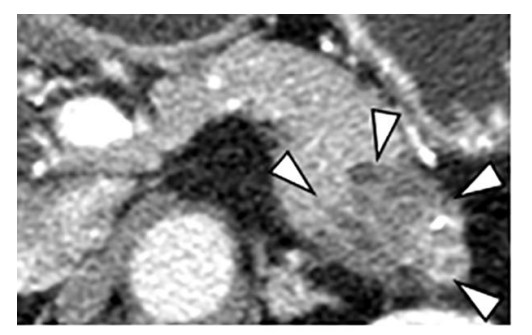

C
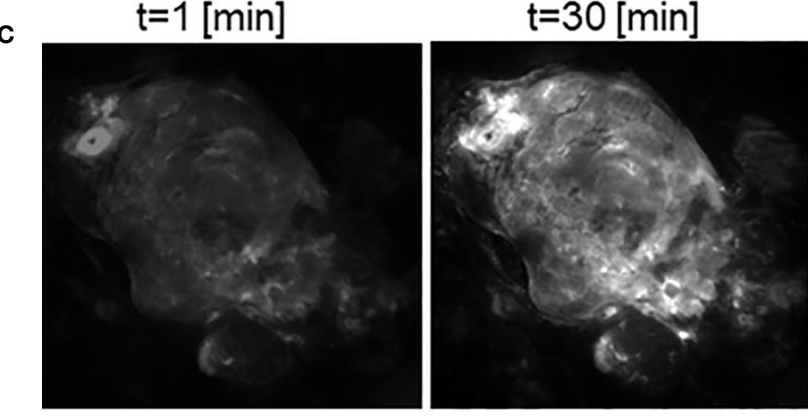

E

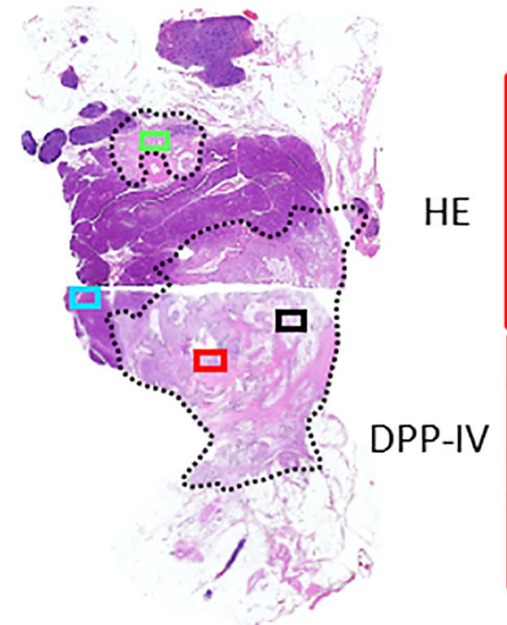

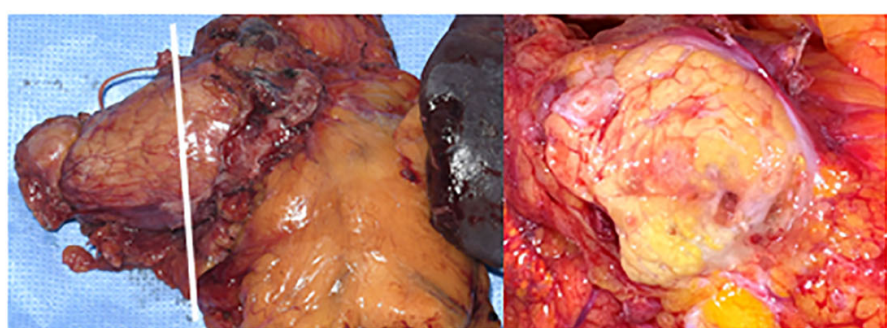

Pseudo-real color
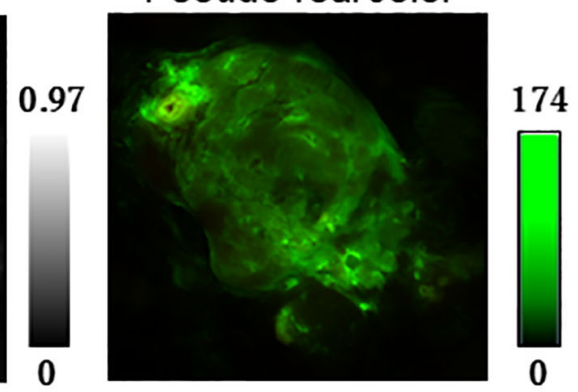
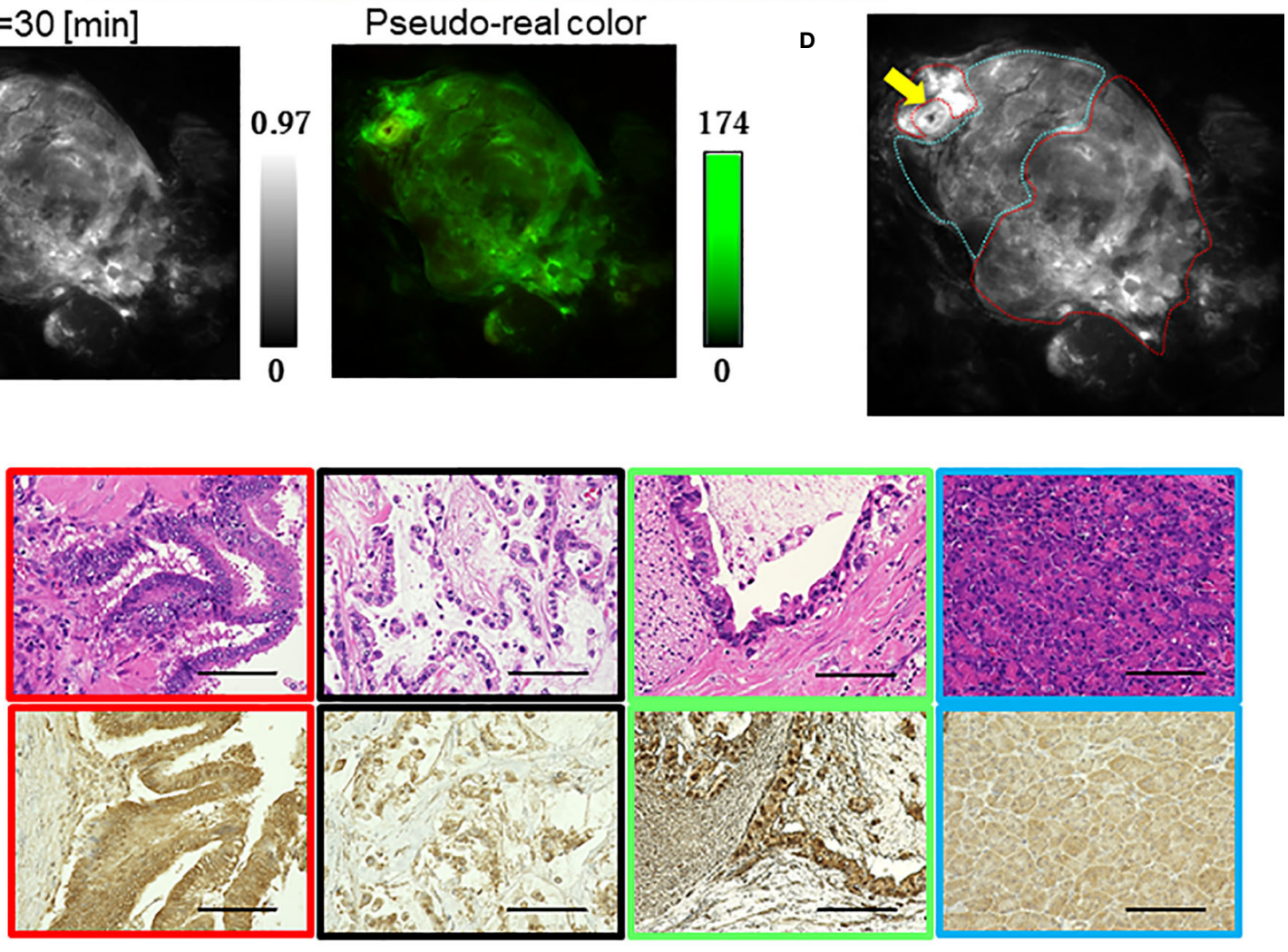

FIGURE 4 | Fluorescence imaging using GP-HMRG to probe whole surgical specimens demonstrates cancer infiltration to the splenic artery (Patient No. 8). (A) Preoperative contrast-enhanced computed tomography of pancreatic body cancer (arrowheads). (B) Macroscopic images of the DP specimen (left) and cut surface along the dotted line including the tumor (right). (C) Increase of fluorescence signals after spraying GP-HMRG on the cut surface and pseudo-real color image at $30 \mathrm{~min}$. (D) Relationships between fluorescence signals and distributions of cancer tissues (red dotted line) and surrounding pancreatic tissues (blue dotted line) according to histological findings. Arrow indicates the splenic artery. (E) Low-magnification histopathological image of H\&E staining corresponding to fluorescence images (left, dotted line indicates cancer boundaries). Magnified views of H\&E staining and IHC analysis of DPP-IV showing fluorescence (red) and little fluorescence (black) emitted by parts of the main tumor, viable cancer infiltration around the splenic artery (green), and noncancerous pancreatic tissues (blue), (right). Scale bar $=100 \mu \mathrm{m}$. 
A

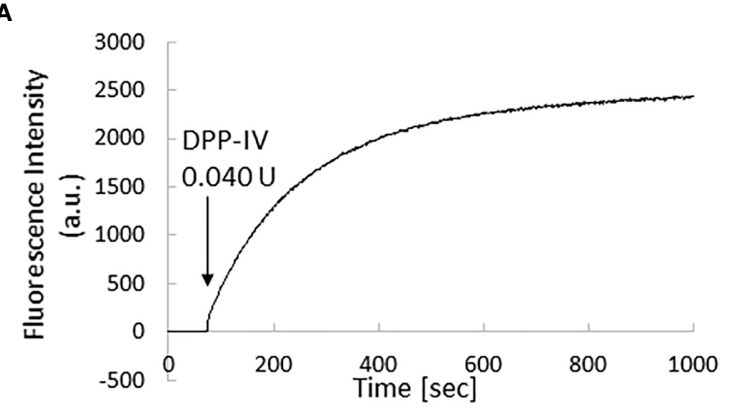

C

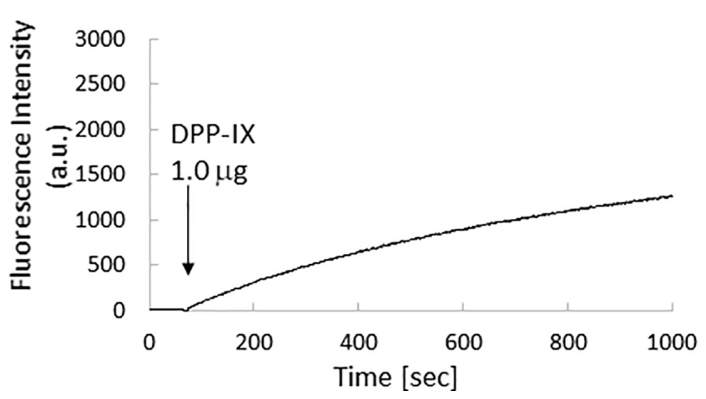

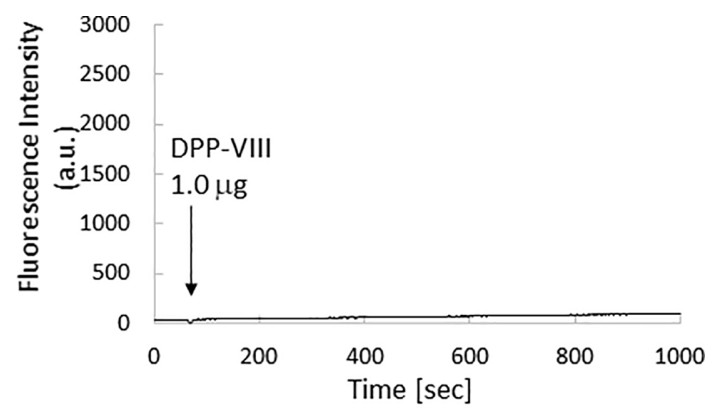

D

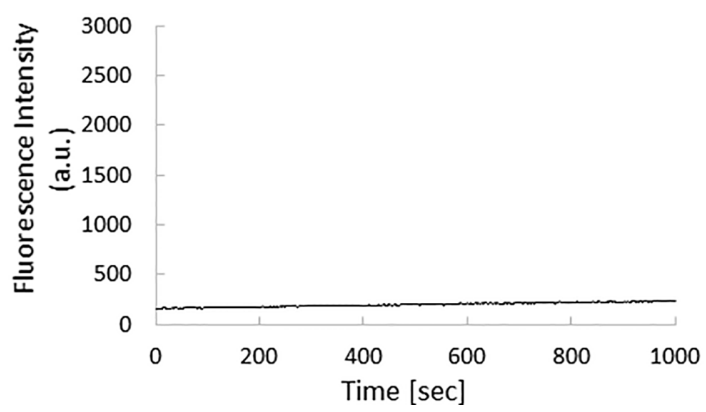

FIGURE 5 | Kinetics of fluorescence intensity of GP-HMRG upon enzyme addition. $\mu$ Excitation/emission wavelengths were $495 \mathrm{~nm} / 525 \mathrm{~nm}$. Trends of fluorescence

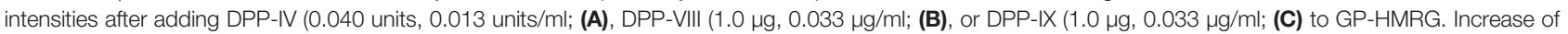
fluorescence intensity of GP-HMRG with no addition of enzymes was also demonstrated in panel (D).

The present technique offers potential advantages for detecting tumor cells with high enzymatic activities specific to cancer tissues, which may facilitate prediction of a patient's sensitivity to chemotherapy and postoperative outcome, as previously suggested in case of fluorescence imaging of colorectal liver metastasis by gGlu-HMRG (17).

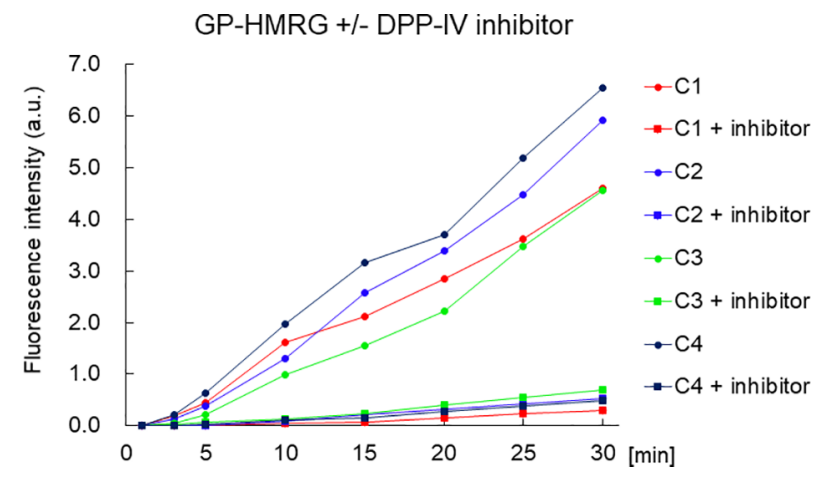

FIGURE 6 | The time course of the increase of Fl of small cancer specimen with or without inhibitor. The fluorescence intensity of the pancreatic cancer specimen was decreased with DPP-IV inhibitor. The concentration of the DPP-IV inhibitor (K579, CalbioChem) was $100 \mu \mathrm{M}$.
The most likely candidate as a target enzyme of GP-HMRG is DPP-IV that cleaves the N-terminal residue of Xaa-Pro/Ala to regulate the bioavailability of glucose-insulinotropic peptide (GIP) and glucagon-like peptide-1 (GLP-1) (39). Other studies demonstrate the upregulation of DPP-IV in malignancies (4042) such as pancreatic cancer (43), although IHC analysis here did not demonstrate unambiguous differences in the expression levels of DPP-IV between cancer tissues and noncancerous tissues, likely because of the different antibodies used to detect DPP-IV or for other technical reasons. Another candidate enzyme is DPP-IX, which is upregulated in pancreatic cancer tissues (43) and cleaves GP-HMRG, although IHC staining of DPP-IX expression sufficient for pathological analysis was unavailable.

The limitation of this study lies in its small sample size. With the sufficient number of lysates and tissue samples for robust statistical analyses, more promising fluorophores other than GPHMRG might have been identified in the initial screening processes. Considering the heterogeneity of pancreatic cancers and factors affecting the enzymatic activities of cancer tissues, we must continue to conduct evaluation of the efficiency of GPHMRG for fluorescence imaging to identify cancer tissue using an observer-blinded trial, as well as to predict postoperative outcomes of a larger population. Furthermore, the potentially insufficient levels of DPP-IV in cancer cells, as well as in stromal and Langerhans islets $(43,44)$, may decrease the sensitivity of cancer detection in certain patients. The expression status of 
DPP-IV and DPP-IX must therefore be evaluated using IHC and the diced electrophoresis gel assay (DEG-Assay) (45).

In conclusion, fluorescence imaging using GP-HMRG may enable rapid and real-time visualization of pancreatic cancer through the detection of cancer tissue-specific enzymatic activities.

\section{DATA AVAILABILITY STATEMENT}

The raw data supporting the conclusions of this article will be made available by the authors, without undue reservation.

\section{ETHICS STATEMENT}

The studies involving human participants were reviewed and approved by The Institutional Review Board of the University of Tokyo Hospital (IRB No. 2957-[11]). The patients/participants provided their written informed consent to participate in this study. Written informed consent was obtained from the individual(s) for the publication of any potentially identifiable images or data included in this article.

\section{AUTHOR CONTRIBUTIONS}

RT, MS, and TI drafted the initial manuscript. RT and MS collected the samples, acquired the data, and performed the analysis. YK and MK prepared a library of activatable fluorescence probes. RT and MT evaluated the pathological extent of tumor. RT and YI performed immunohistochemistry,

\section{REFERENCES}

1. Hidalgo M. Pancreatic Cancer. N Engl J Med (2010) 362(17):1605-17. doi: 10.1056/NEJMra0901557

2. Siegel RL, Miller KD, Jemal A. Cancer Statistics, 2020. CA Cancer J Clin (2020) 70(1):7-30. doi: 10.3322/caac.21590

3. Ferlay J, Colombet M, Soerjomataram I, Dyba T, Randi G, Bettio M, et al. Cancer Incidence and Mortality Patterns in Europe: Estimates for 40 Countries and 25 Major Cancers in 2018. Eur J Cancer (2018) 103:356-87. doi: 10.1016/j.ejca.2018.07.005

4. Chen W, Zheng R, Baade PD, Zhang S, Zeng H, Bray F, et al. Cancer Statistics in China, 2015. CA Cancer J Clin (2016) 66(2):115-32. doi: 10.3322/caac.21338

5. Wagner M, Redaelli C, Lietz M, Seiler CA, Friess H, Büchler MW. Curative Resection is the Single Most Important Factor Determining Outcome in Patients With Pancreatic Adenocarcinoma. Br J Surg (2004) 91(5):586-94. doi: $10.1002 /$ bjs.4484

6. Demir IE, Jäger C, Schlitter AM, Konukiewitz B, Stecher L, Schorn S, et al. R0 Versus R1 Resection Matters After Pancreaticoduodenectomy, and Less After Distal or Total Pancreatectomy for Pancreatic Cancer. Ann Surg (2018) 268 (6):1058-68. doi: 10.1097/SLA.0000000000002345

7. Tummers WS, Groen JV, Sibinga Mulder BG, Farina-Sarasqueta A, Morreau J, Putter H, et al. Impact of Resection Margin Status on Recurrence and Survival in Pancreatic Cancer Surgery. Br J Surg (2019) 106(8):1055-65. doi: 10.1002/bjs.11115

8. Gillen S, Schuster T, Meyer Zum Büschenfelde C, Friess H, Kleeff J. Preoperative/neoadjuvant Therapy in Pancreatic Cancer: A Systematic Review and Meta-Analysis of Response and Resection Percentages. PloS Med (2010) 7(4):e1000267. doi: 10.1371/journal.pmed.1000267 and MT evaluated the results. MK, TU, YU, and $\mathrm{KH}$ critically evaluated and revised the manuscript. All authors contributed to the article and approved the submitted version.

\section{FUNDING}

This work was supported by collaborative research funding between The University of Tokyo and NIPRO Corporation, and grants from the Ministry of Education, Culture, Sports, Science and Technology of Japan [Grant Number 19H05632 to $\mathrm{YU}$. The funder was not involved in the study design, collection, analysis, interpretation of data, the writing of this article or the decision to submit it for publication.

\section{ACKNOWLEDGMENTS}

We thank Drs. Yoshikuni Kawaguchi, Akihiko Ichida, Nobuhisa Akamatsu, Junichi Kaneko, and Junichi Arita for obtaining patients' informed consent; Dr. Eiichi Yasunaga for supporting sample collection; and Edanz Group (https://www.jp.edanz.com/ ac) for editing a draft of this manuscript.

\section{SUPPLEMENTARY MATERIAL}

The Supplementary Material for this article can be found online at: https://www.frontiersin.org/articles/10.3389/fonc.2021. 714527/full\#supplementary-material

9. Lutfi W, Talamonti MS, Kantor O, Wang CH, Liederbach E, Stocker SJ, et al. Perioperative Chemotherapy is Associated With a Survival Advantage in Early Stage Adenocarcinoma of the Pancreatic Head. Surgery (2016) 160 (3):714-24. doi: 10.1016/j.surg.2016.05.029

10. Verbeke C, Löhr M, Karlsson JS, Del Chiaro M. Pathology Reporting of Pancreatic Cancer Following Neoadjuvant Therapy: Challenges and Uncertainties. Cancer Treat Rev (2015) 41(1):17-26. doi: 10.1016/j.ctrv.2014.11.002

11. Urano Y, Sakabe M, Kosaka N, Ogawa M, Mitsunaga M, Asanuma D, et al. Rapid Cancer Detection by Topically Spraying a $\gamma$-GlutamyltranspeptidaseActivated Fluorescent Probe. Sci Transl Med (2011) 3(110):110ra119. doi: 10.1126/scitranslmed.3002823

12. Fujita K, Kamiya M, Yoshioka T, Ogasawara A, Hino R, Kojima R, et al. Rapid and Accurate Visualization of Breast Tumors With a Fluorescent Probe Targeting $\alpha$-Mannosidase 2c1. ACS Cent Sci (2020) 6(12):2217-27. doi: 10.1021/acscentsci.0c01189

13. Ueo H, Shinden Y, Tobo T, Gamachi A, Udo M, Komatsu H, et al. Rapid Intraoperative Visualization of Breast Lesions With $\gamma$-Glutamyl Hydroxymethyl Rhodamine Green. Sci Rep (2015) 5:12080. doi: 10.1038/srep12080

14. Shinden Y, Ueo H, Tobo T, Gamachi A, Utou M, Komatsu H, et al. Rapid Diagnosis of Lymph Node Metastasis in Breast Cancer Using a New Fluorescent Method With $\gamma$-Glutamyl Hydroxymethyl Rhodamine Green. Sci Rep (2016) 6:27525. doi: 10.1038/srep27525

15. Onoyama H, Kamiya M, Kuriki Y, Komatsu T, Abe H, Tsuji Y, et al. Rapid and Sensitive Detection of Early Esophageal Squamous Cell Carcinoma With Fluorescence Probe Targeting Dipeptidylpeptidase IV. Sci Rep (2016) 6:26399. doi: 10.1038/srep26399

16. Yamamoto K, Ohnishi S, Mizushima T, Kodaira J, Ono M, Hatanaka Y, et al. Detection of Early Adenocarcinoma of the Esophagogastric Junction by 
Spraying an Enzyme-Activatable Fluorescent Probe Targeting Dipeptidyl peptidase-IV. BMC Cancer (2020) 20(1):64. doi: 10.1186/s12885-020-6537-9

17. Miyata Y, Ishizawa T, Kamiya M, Yamashita S, Hasegawa K, Ushiku A, et al. Intraoperative Imaging of Hepatic Cancers Using $\gamma$-GlutamyltranspeptidaseSpecific Fluorophore Enabling Real-Time Identification and Estimation of Recurrence. Sci Rep (2017) 7(1):3542. doi: 10.1038/s41598-017-03760-3

18. Hino H, Kamiya M, Kitano K, Mizuno K, Tanaka S, Nishiyama N, et al. Rapid Cancer Fluorescence Imaging Using A $\gamma$-Glutamyltranspeptidase-Specific Probe For Primary Lung Cancer. Transl Oncol (2016) 9(3):203-10. doi: 10.1016/j.tranon.2016.03.007

19. Mizushima T, Ohnishi S, Shimizu Y, Hatanaka Y, Hatanaka KC, Hosono H, et al. Fluorescent Imaging of Superficial Head and Neck Squamous Cell Carcinoma Using a $\gamma$-Glutamyltranspeptidase-Activated Targeting Agent: A Pilot Study. BMC Cancer (2016) 16:411. doi: 10.1186/s12885-016-2421-Z

20. Mizushima T, Ohnishi S, Shimizu Y, Hatanaka Y, Hatanaka KC, Kuriki Y, et al. Rapid Detection of Superficial Head and Neck Squamous Cell Carcinoma by Topically Spraying Fluorescent Probe Targeting Dipeptidyl peptidase-IV. Head Neck (2018) 40(7):1466-75. doi: 10.1002/hed.25126

21. Sato C, Abe S, Saito Y, So Tsuruki E, Takamaru H, Makazu M, et al. A Pilot Study of Fluorescent Imaging of Colorectal Tumors Using a $\gamma$-GlutamylTranspeptidase-Activatable Fluorescent Probe. Digestion (2015) 91(1):70-6. doi: $10.1159 / 000369367$

22. Hino R, Inoshita N, Yoshimoto T, Ogawa M, Miura D, Watanabe R, et al. Rapid Detection of Papillary Thyroid Carcinoma by Fluorescence Imaging Using a $\gamma$-Glutamyltranspeptidase-Specific Probe: A Pilot Study. Thyroid Res (2018) 11:16. doi: 10.1186/s13044-018-0060-y

23. Kitagawa Y, Tanaka S, Kamiya M, Kuriki Y, Yamamoto K, Shimizu T, et al. A Novel Topical Fluorescent Probe for Detection of Glioblastoma. Clin Cancer Res (2021)27(14):3936-47. doi: 10.1158/1078-0432

24. Eser S, Messer M, Eser P, von Werder A, Seidler B, Bajbouj M, et al. In Vivo Diagnosis of Murine Pancreatic Intraepithelial Neoplasia and Early-Stage Pancreatic Cancer by Molecular Imaging. Proc Natl Acad Sci U S A (2011) 108 (24):9945-50. doi: 10.1073/pnas.1100890108

25. Cruz-Monserrate Z, Abd-Elgaliel WR, Grote T, Deng D, Ji B, Arumugam T, et al. Detection of Pancreatic Cancer Tumours and Precursor Lesions by Cathepsin E Activity in Mouse Models. Gut (2012) 61(9):1315-22. doi: 10.1136/gutjnl-2011-300544

26. Li H, Li Y, Cui L, Wang B, Cui W, Li M, et al. Monitoring Pancreatic Carcinogenesis by the Molecular Imaging of Cathepsin E In Vivo Using Confocal Laser Endomicroscopy. PloS One (2014) 9(9):e106566. doi: 10.1371/ journal.pone. 0106566

27. Harada K, Murayama Y, Kubo H, Matsuo H, Morimura R, Ikoma H, et al. Photodynamic Diagnosis of Peritoneal Metastasis in Human Pancreatic Cancer Using 5-Aminolevulinic Acid During Staging Laparoscopy. Oncol Lett (2018) 16(1):821-8. doi: 10.3892/ol.2018.8732

28. Newton AD, Predina JD, Shin MH, Frenzel-Sulyok LG, Vollmer CM, Drebin JA, et al. Intraoperative Near-Infrared Imaging Can Identify Neoplasms and Aid in Real-Time Margin Assessment During Pancreatic Resection. Ann Surg (2019) 270(1):12-20. doi: 10.1097/SLA.0000000000003201

29. McElroy M, Kaushal S, Luiken GA, Talamini MA, Moossa AR, Hoffman RM, et al. Imaging of Primary and Metastatic Pancreatic Cancer Using a Fluorophore-Conjugated Anti-CA19-9 Antibody for Surgical Navigation. World J Surg (2008) 32(6):1057-66. doi: 10.1007/s00268-007-9452-1

30. Kaushal S, McElroy MK, Luiken GA, Talamini MA, Moossa AR, Hoffman $\mathrm{RM}$, et al. Fluorophore-Conjugated Anti-CEA Antibody for the Intraoperative Imaging of Pancreatic and Colorectal Cancer. J Gastrointest Surg (2008) 12 (11):1938-50. doi: 10.1007/s11605-008-0581-0

31. Lwin TM, Murakami T, Miyake K, Yazaki PJ, Shivley JE, Hoffman RM, et al. Tumor-Specific Labeling of Pancreatic Cancer Using a Humanized Anti-CEA Antibody Conjugated to a Near-Infrared Fluorophore. Ann Surg Oncol (2018) 25(4):1079-85. doi: 10.1245/s10434-018-6344-6

32. Lwin TM, Miyake K, Murakami T, DeLong JC, Amirfakhri S, Filemoni F, et al. Fluorescent Humanized Anti-CEA Antibody Specifically Labels Metastatic Pancreatic Cancer in a Patient-Derived Orthotopic Xenograft (PDOX) Mouse Model. Oncotarget (2018) 9(99):37333-42. doi: 10.18632/ oncotarget.26484

33. Hiroshima Y, Maawy A, Sato S, Murakami T, Uehara F, Miwa S, et al. HandHeld High-Resolution Fluorescence Imaging System for Fluorescence-Guided
Surgery of Patient and Cell-Line Pancreatic Tumors Growing Orthotopically in Nude Mice. J Surg Res (2014) 187(2):510-7. doi: 10.1016/j.jss.2013.11.1083 34. Hiroshima Y, Maawy A, Zhang Y, Murakami T, Momiyama M, Mori R, et al. Fluorescence-Guided Surgery, But Not Bright-Light Surgery, Prevents Local Recurrence in a Pancreatic Cancer Patient Derived Orthotopic Xenograft (PDOX) Model Resistant to Neoadjuvant Chemotherapy (NAC). Pancreatology (2015) 15(3):295-301. doi: 10.1016/j.pan.2015.02.008

35. Hoogstins CES, Boogerd LSF, Sibinga Mulder BG, Mieog JSD, Swijnenburg $\mathrm{RJ}$, van de Velde $\mathrm{CJH}$, et al. Image-Guided Surgery in Patients With Pancreatic Cancer: First Results of a Clinical Trial Using SGM-101, a Novel Carcinoembryonic Antigen-Targeting, Near-Infrared Fluorescent Agent. Ann Surg Oncol (2018) 25(11):3350-7. doi: 10.1245/s10434-018-6655-7

36. Tummers WS, Miller SE, Teraphongphom NT, Gomez A, Steinberg I, Huland DM, et al. Intraoperative Pancreatic Cancer Detection Using Tumor-Specific Multimodality Molecular Imaging. Ann Surg Oncol (2018) 25(7):1880-8. doi: 10.1245/s10434-018-6453-2

37. Tummers WS, Miller SE, Teraphongphom NT, van den Berg NS, Hasan A, Longacre TA, et al. Detection of Visually Occult Metastatic Lymph Nodes Using Molecularly Targeted Fluorescent Imaging During Surgical Resection of Pancreatic Cancer. HPB (Oxford) (2019) 21(7):883-90. doi: 10.1016/ j.hpb.2018.11.008

38. Park JY, Lee JY, Zhang Y, Hoffman RM, Bouvet M. Targeting the Insulin Growth Factor-1 Receptor With Fluorescent Antibodies Enables High Resolution Imaging of Human Pancreatic Cancer in Orthotopic Mouse Models. Oncotarget (2016) 7(14):18262-8. doi: 10.18632/oncotarget.7576

39. Lambeir AM, Durinx C, Scharpé S, De Meester I. Dipeptidyl-Peptidase IV From Bench to Bedside: An Update on Structural Properties, Functions, and Clinical Aspects of the Enzyme DPP Iv. Crit Rev Clin Lab Sci (2003) 40 (3):209-94. doi: 10.1080/713609354

40. Havre PA, Abe M, Urasaki Y, Ohnuma K, Morimoto C, Dang NH. The Role of CD26/dipeptidyl Peptidase IV in Cancer. Front Biosci (2008) 13:1634-45. doi: $10.2741 / 2787$

41. Cordero OJ, Salgado FJ, Nogueira M. On the Origin of Serum CD26 and its Altered Concentration in Cancer Patients. Cancer Immunol Immunother (2009) 58(11):1723-47. doi: 10.1007/s00262-009-0728-1

42. Ghersi G, Zhao Q, Salamone M, Yeh Y, Zucker S, Chen WT. The Protease Complex Consisting of Dipeptidyl Peptidase IV and Seprase Plays a Role in the Migration and Invasion of Human Endothelial Cells in Collagenous Matrices. Cancer Res (2006) 66(9):4652-61. doi: 10.1158/0008-5472.CAN-05-1245

43. Busek P, Vanickova Z, Hrabal P, Brabec M, Fric P, Zavoral M, et al. Increased Tissue and Circulating Levels of Dipeptidyl Peptidase-IV Enzymatic Activity in Patients With Pancreatic Ductal Adenocarcinoma. Pancreatology (2016) 16 (5):829-38. doi: 10.1016/j.pan.2016.06.001

44. Busek P, Hrabal P, Fric P, Sedo A. Co-Expression of the Homologous Proteases Fibroblast Activation Protein and Dipeptidyl Peptidase-IV in the Adult Human Langerhans Islets. Histochem Cell Biol (2015) 143(5):497-504. doi: 10.1007/s00418-014-1292-0

45. Komatsu T, Hanaoka K, Adibekian A, Yoshioka K, Terai T, Ueno T, et al. Diced Electrophoresis Gel Assay for Screening Enzymes With Specified Activities. J Am Chem Soc (2013) 135(16):6002-5. doi: 10.1021/ja401792d

Conflict of Interest: The authors declare that the research was conducted in the absence of any commercial or financial relationships that could be construed as a potential conflict of interest.

Publisher's Note: All claims expressed in this article are solely those of the authors and do not necessarily represent those of their affiliated organizations, or those of the publisher, the editors and the reviewers. Any product that may be evaluated in this article, or claim that may be made by its manufacturer, is not guaranteed or endorsed by the publisher.

Copyright (๑) 2021 Takahashi, Ishizawa, Sato, Inagaki, Takanka, Kuriki, Kamiya, Ushiku, Urano and Hasegawa. This is an open-access article distributed under the terms of the Creative Commons Attribution License (CC BY). The use, distribution or reproduction in other forums is permitted, provided the original author(s) and the copyright owner(s) are credited and that the original publication in this journal is cited, in accordance with accepted academic practice. No use, distribution or reproduction is permitted which does not comply with these terms. 